\title{
Aminas bioativas e características físico-químicas de salames tipo italiano
}

[Bioactive amines and physico-chemical characteristics of Italian sausages]

\author{
J. Caccioppoli, F.B. Custódio, S.M. Vieira, J.V. Coelho, M.B.A. Glória* \\ Faculdade de Farmácia - UFMG \\ Av. Antonio Carlos. 6627 \\ 31270-901 - Belo Horizonte, MG
}

\begin{abstract}
RESUMO
Amostras de seis lotes de sete marcas de salames tipo italiano foram analisadas quanto a aminas bioativas e características físico-químicas de qualidade. Oito das 10 aminas pesquisadas foram detectadas em todas as amostras com teores totais de 28,33 a $53,27 \mathrm{mg} / 100 \mathrm{~g}$. A tiramina foi a amina predominante seguida da putrescina e da cadaverina. Todas as marcas apresentaram teores de tiramina capazes de causar efeito tóxico em indivíduos sensíveis dependendo da quantidade ingerida. Duas marcas apresentaram também teores potencialmente tóxicos de histamina e de feniletilamina. Nenhuma das marcas atendeu à legislação em pelo menos um parâmetro físico-químico. Os teores de umidade e de açucares totais foram as características com maior percentual de não-atendimento. Os teores de cinzas variaram de 5,54 a $7,12 \mathrm{~g} / 100 \mathrm{~g}$, sendo os cloretos 66 a 79\% desses teores. As amostras apresentaram valores de $\mathrm{pH}$ de 4,86 a 5,78 , acidez de 16,61 a $55,03 \mathrm{ml} \mathrm{NaOH} \mathrm{N} / 100 \mathrm{~g}$ e índice de peróxidos de não detectado a 334,82mEq/kg.
\end{abstract}

Palavras-chave: poliaminas, aminas biogênicas, salame italiano, qualidade

\begin{abstract}
Six batches of seven brands of Italian sausages purchased in Belo Horizonte, MG were analyzed for bioactive amines and physico-chemical characteristics. Eight out of 10 amines investigated were detected in every sample with total levels varying from 28.33 to $53.27 \mathrm{mg} / 100 \mathrm{~g}$. Tyramine was the predominant amine followed by putrescine and cadaverine. Every brand contained toxic tyramine levels for sensitive individuals depending on the amount of sausage consumption. Two brands also contained toxic levels of histamine and phenylethylamine. No brand was in conformity to legislation levels for at least one physico-chemical parameter. The highest discrepancies to legislation levels were observed for moisture and total sugar contents. The levels of ash varied from 5.54 to $7.12 \mathrm{~g} / 100 \mathrm{~g}$, with chlorides representing 66 to $79 \%$ of the levels. The $\mathrm{pH}$ varied from 4.86 to 5.78 , acidity from 16.61 to $55.03 \mathrm{ml} \mathrm{NaOH} \mathrm{N/100g}$ and peroxide values from 0.0 (no detected) to $334.82 \mathrm{mEq} / \mathrm{kg}$.
\end{abstract}

Keywords: polyamines, biogenic amines, Italian sausage, quality

\section{INTRODUÇÃO}

Vários tipos de salames são produzidos no Brasil, os quais variam em função da composição, calibre, grau de moagem dos ingredientes, especiarias e condimentos adicionados, defumação ou não e período de maturação. $\mathrm{O}$ tipo italiano, um dos mais populares no país, caracteriza-se por moagem média (6 a 9mm), é curado, fermentado, apresenta tempo de maturação de 25 a 50 dias e é dessecado (Instrução..., 2000).

Recebido em 20 de setembro de 2005

Aceito em 15 de maio de 2006

* Autor para correspondência (corresponding author)

E-mail: beatriz@farmacia.ufmg.br 
A qualidade de salames é avaliada em função dos teores máximos permitidos de umidade, atividade de água, lipídios e açúcares totais e dos teores mínimos exigidos de proteínas. No caso do salame tipo italiano, esses valores são, respectivamente, $35 \mathrm{~g} / 100 \mathrm{~g} ; \quad 0,90 ; \quad 32 \mathrm{~g} / 100 \mathrm{~g}$; $1,5 \mathrm{~g} / 100 \mathrm{~g} ;$ e $25 \mathrm{~g} / 100 \mathrm{~g}$ (Instrução..., 2000). Na Holanda e na República Tcheca, são também estipulados limites máximos para histamina de 10 e $20 \mathrm{mg} / 100 \mathrm{~g}$, respectivamente (Montel et al., 1999).

A histamina faz parte de um grupo de substâncias denominadas aminas bioativas, as quais desempenham papel importante no metabolismo celular. Entretanto, podem causar efeitos tóxicos quando presentes nos alimentos em concentrações elevadas ou quando a atividade das enzimas metabolizadoras dessas substâncias no homem for deficiente por problemas genéticos ou inibida por agentes farmacológicos. As intoxicações alimentares mais freqüentes causadas por aminas envolvem a histamina, a tiramina e a feniletilamina (Fuzikawa et al., 1999; Glória, 2005).

Sob condições fisiológicas normais, os músculos contêm as poliaminas espermina e espermidina as quais atuam como hormônio ou fator de crescimento, sendo essenciais para as células. A putrescina é um intermediário obrigatório na formação das poliaminas (Silva e Glória, 2002; Vasconcelos-Neto, 2003; Glória, 2005). Outras aminas, as biogênicas, podem ser formadas por microrganismos que possuem atividade aminoácido-descarboxilase, sejam estes adicionados intencionalmente (cultura iniciadora), ou não (microbiota contaminante). Tendo em vista os processos de fermentação e maturação a que os salames são submetidos, esses se tornam susceptíveis à formação de aminas. De fato, a presença de tiramina e histamina em teores elevados tem sido relatada em produtos cárneos fermentados ou maturados de vários países (Eerola et al., 1997; HernándezJover et al., 1997a; Montel et al., 1999; BoverCid et al., 2000a, Suzzi e Gardini, 2003).

São poucos os estudos sobre aminas em produtos cárneos brasileiros. Existem apenas dados sobre os teores de histamina em algumas amostras de lingüiça, presunto e salame (Leitão et al., 1983) e sobre os teores de aminas na carne e em produtos à base de frango: salsicha, mortadela, lingüiça frescal, almôndega, hambúrguer e empanado (Silva e Glória, 2002).

Este trabalho teve como objetivos avaliar a qualidade de salames do tipo italiano comercializados em Belo Horizonte, MG, comparar os ingredientes descritos nos rótulos, determinar os tipos e os teores de aminas bioativas e as características físico-químicas, e estimar a correlação entre as características analisadas.

\section{MATERIAL E MÉTODOS}

Foram adquiridas no mercado consumidor de Belo Horizonte, MG, entre agosto de 2001 a maio de 2002, amostras de salames do tipo italiano com selo do Serviço de Inspeção Federal. Foram adquiridas seis amostras de salames de lotes diferentes de sete marcas, num total de 42 amostras. Todos os reagentes utilizados nas análises eram de grau p.a., exceto os solventes para as análises cromatográficas. As aminas utilizadas como padrão - dihidrocloreto de putrescina, dihidrocloreto de cadaverina, tiramina, dihidrocloreto de histamina, hidrocloreto de serotonina, sulfato de agmatina, trihidrocloreto de espermidina, tetrahidrocloreto de espermina, dihidrocloreto de 2-feniletilamina e triptamina - foram adquiridas da Sigma ${ }^{1}$.

Cada amostra de salame $(\sim 300 \mathrm{~g})$ foi triturada em processador de alimentos e homogeneizada. Todas as análises foram feitas em duplicata e dentro dos prazos de validade estipulados nos rótulos. As aminas bioativas foram extraídas com ácido tricloroacético a 5\%, separadas por CLAEpar iônico e quantificadas fluorimetricamente a $340 \mathrm{~nm}$ de excitação e $445 \mathrm{~nm}$ de emissão (Silva e Glória, 2002). As amostras foram também analisadas quanto a atividade de água, umidade, lipídios, proteínas e açúcares totais (Instrução..., 2000). Além desses parâmetros, foram feitas análises de nitrito e nitrato de sódio (Elliot e Porter, 1974), pH (Normas..., 1985), acidez na gordura, índice de peróxidos, cinzas e cloretos (Instrução..., 1999).

\footnotetext{
${ }^{1}$ Sigma Aldrich, St Louis, Missouri, USA
} 
Os resultados obtidos foram submetidos à análise de variância, e as médias comparadas pelo teste Tukey a 5\% de significância. Estimou-se a correlação de Pearson em nível de $1 \%$ de significância entre as aminas, e entre as aminas e as caracteristicas físico-químicas.

\section{RESULTADOS E DISCUSSÃO}

Ao avaliar os rótulos das amostras de salame, observou-se a utilização dos ingredientes opcionais carne bovina e leite em pó em $57 \%$; açúcares em $43 \%$, e maltodextrina em $14 \%$ das amostras. Vários tipos de condimentos foram declarados como especiarias, condimentos e aromatizantes naturais, pimentas branca, preta e vermelha e vinho branco. Dentre os aditivos permitidos (Portaria..., 1998), o glutamato de sódio foi declarado em $14 \%$ das amostras, o nitrito e o nitrato de sódio em $71 \%$ e $86 \%$ das amostras, respectivamente, sendo o uso concomitante destes relatado em $57 \%$ das amostras. $\mathrm{O}$ eritorbato de sódio foi declarado em $71 \%$ e o ácido cítrico em $14 \%$ das amostras. Apenas duas indústrias declararam o uso de culturas iniciadoras, apesar dos benefícios que podem trazer para o produto, dentre eles, a redução do $\mathrm{pH}$, o direcionamento da microbiota dominante impedindo a prevalência de contaminantes, e o desenvolvimento do flavor e textura do produto (Hernandez-Jover et al., 1997b; Ayhan et al., 1999; Bover-Cid et al., 2000b).

Com exceção da serotonina e agmatina, que foram encontradas em, respectivamente, $14 \mathrm{e}$ $71 \%$ das amostras analisadas, as demais aminas foram detectadas em todas as amostras. A presença destas já havia sido relatada em diversos tipos de salames como fuet, salchichón, chorizo e sobrasada, salames egípcios, canadenses, finlandeses, americanos e italianos (Eerola et al., 1997; Hernández-Jover et al., 1997a; Bover-Cid et al., 2000a; Suzzi e Gardini, 2003; Coisson et al., 2004). A presença das poliaminas espermina e espermidina era esperada uma vez que são inerentes ao tecido animal (Hernández-Jover et al., 1997a; Suzzi e Gardini, 2003; Glória, 2005). As demais aminas, as biogênicas, podem ter sido introduzidas pelos ingredientes adicionados ou formadas durante o processamento (Eerola et al., 1997; Suzzi e Gardini, 2003; Glória, 2005).
Na Fig. 1, observa-se a contribuição de cada amina ao teor total nas diferentes marcas de salame. As aminas biogênicas contribuíram com maior percentual comparado com as poliaminas. As poliaminas são as aminas predominantes no tecido cárneo fresco (Hernández-Jover et al., 1997a; Silva e Glória, 2002; Vasconcelos-Neto, 2003). Entretanto, o perfil foi alterado nas amostras de salame. A espermina representou apenas 6 a 14\%, e a espermidina não teve contribuição significativa $(<0,9 \%)$ ao teor total de aminas. Com relação às aminas biogênicas, a tiramina foi a que mais contribuiu ao teor total $(34,1$ a $51,8 \%)$ em todas as marcas de salame. A putrescina foi a segunda amina predominante $(17,4$ a $26,7 \%)$ seguida da cadaverina $(1,8$ a $24,6 \%$ ) na maioria das marcas. A histamina contribuiu com $\leq 6,1 \%$, exceto na marca $G$ que continha $32,7 \%$. As demais aminas representaram pequena fração do teor total: a triptamina até 3,1\%, a feniletilamina até 5,8\%, e a agmatina até $1,7 \%$. A contribuição da serotonina foi insignificante (não incluída na Fig. 1). A predominância da tiramina sobre a histamina em salames também foi descrita na literatura (Eerola et al., 1997; Hernández-Jover et al., 1997a,b; Montel et al., 1999; Suzzi e Gardini, 2003; Coisson et al., 2004).

Os teores de aminas estão indicados na Tab. 1. Os teores individuais e totais de aminas variaram significativamente entre marcas e entre amostras de uma mesma marca. Diversos autores também relataram grande variabilidade nos teores de aminas em produtos cárneos (Eerola et al., 1997, Hernández-Jover et al., 1997a; Silva e Glória, 2002; Suzzi e Gardini, 2003). A variação entre marcas é possível uma vez que cada indústria tem sua formulação e sistema de processamento. Além disso, fatores como a qualidade higiênicosanitária e as condições de descongelamento das matérias primas, o tipo de cultura iniciadora utilizada, as etapas do processamento (temperaturas de fermentação, maturação e armazenamento), e as condições higiênicosanitárias durante o processamento podem afetar os teores de aminas (Hernández-Jover et al., 1997a; Bover-Cid et al., 2000a). Esses fatores constituem os pontos críticos para controle da formação de aminas. 

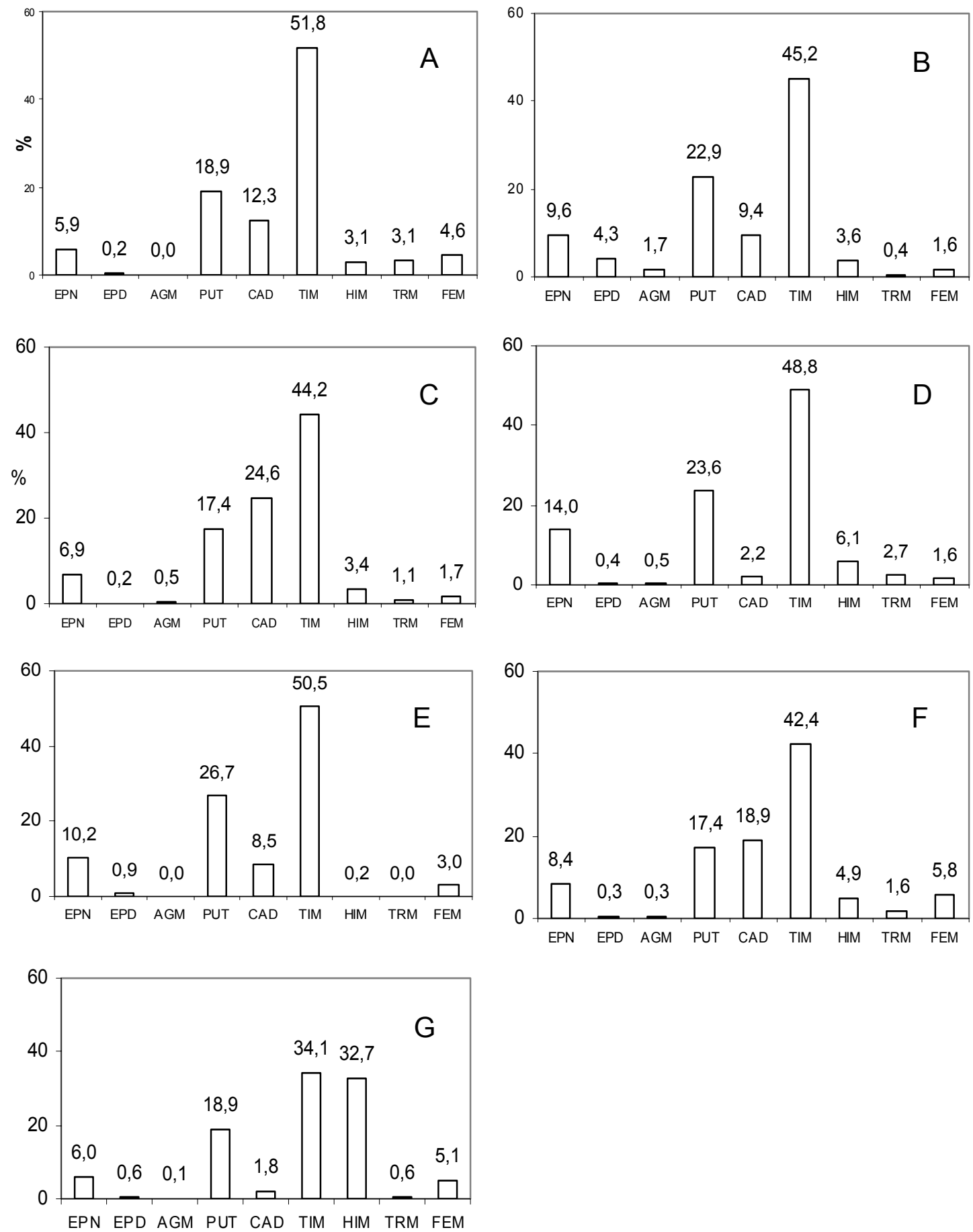

Figura 1. Percentual de contribuição individual das aminas bioativas ao teor total em diferentes marcas de salame tipo italiano comercializadas em Belo Horizonte, $\mathrm{MG}$, no período de agosto de 2001 a maio de 2002 (EPN = espermina, EPD = espermidina, AGM = agmatina, PUT = putrescina, $\mathrm{CAD}=$ cadaverina, $\mathrm{TIM}=$ tiramina, $\mathrm{HIM}=$ histamina, $\mathrm{TRM}=$ triptamina, $\mathrm{FEM}=$ feniletilamina). 
Tabela 1. Teores médios de aminas bioativas em diferentes marcas de salame tipo italiano comercializadas em Belo Horizonte, MG, no período de agosto de 2001 a maio de 2002

\begin{tabular}{lccccccc}
\hline \multirow{2}{*}{ Amina } & \multicolumn{7}{c}{ Teor médio (desvio-padrão) $\mathrm{em} \mathrm{mg} / 100 \mathrm{~g}$} \\
\cline { 2 - 8 } Espermina & $\mathrm{A}$ & $\mathrm{B}$ & $\mathrm{C}$ & $\mathrm{D}$ & $\mathrm{E}$ & $\mathrm{F}$ & $\mathrm{G}$ \\
\hline \multirow{2}{*}{ Espermidina } & $1,67 \mathrm{~d}$ & $3,72 \mathrm{c}$ & $3,61 \mathrm{c}$ & $5,93 \mathrm{a}$ & $4,52 \mathrm{~b}$ & $4,48 \mathrm{~b}, \mathrm{c}$ & $2,23 \mathrm{~d}$ \\
& $(0,118)$ & $(0,748)$ & $(0,350)$ & $(0,923)$ & $(0,472)$ & $(0,416)$ & $(0,291)$ \\
\hline \multirow{2}{*}{ Agmatina } & $0,07 \mathrm{~b}$ & $1,67 \mathrm{a}$ & $0,10 \mathrm{~b}$ & $0,16 \mathrm{~b}$ & $0,41 \mathrm{~b}$ & $0,18 \mathrm{~b}$ & $0,22 \mathrm{~b}$ \\
& $(0,003)$ & $(0,867)$ & $(0,003)$ & $(0,043)$ & $(0,047)$ & $(0,007)$ & $(0,004)$ \\
\hline \multirow{2}{*}{ Putrescina } & $0,00 \mathrm{c}$ & $0,67 \mathrm{a}$ & $0,25 \mathrm{~b}$ & $0,21 \mathrm{bc}$ & $0,00 \mathrm{c}$ & $0,14 \mathrm{bc}$ & $0,05 \mathrm{bc}$ \\
& $(0,000)$ & $(0,163)$ & $(0,164)$ & $(0,250)$ & $(0,000)$ & $(0,013)$ & $(0,002)$ \\
\hline \multirow{2}{*}{ Cadaverina } & $5,33 \mathrm{~d}$ & $8,81 \mathrm{bc}$ & $9,04 \mathrm{abc}$ & $9,98 \mathrm{ab}$ & $11,82 \mathrm{a}$ & $9,28 \mathrm{abc}$ & $6,96 \mathrm{c}$ \\
& $(0,957)$ & $(2,744)$ & $(0,783)$ & $(1,831)$ & $(0,746)$ & $(1,261)$ & $(1,606)$ \\
\hline \multirow{2}{*}{ Tiramina } & $3,47 \mathrm{~cd}$ & $3,64 \mathrm{c}$ & $12,81 \mathrm{a}$ & $0,93 \mathrm{~d}$ & $3,79 \mathrm{c}$ & $10,06 \mathrm{~b}$ & $0,68 \mathrm{e}$ \\
& $(1,804)$ & $(1,54)$ & $(1,333)$ & $(0,246)$ & $(2,324)$ & $(1,301)$ & $(0,781)$ \\
\hline \multirow{2}{*}{ Histamina } & $14,60 \mathrm{c}$ & $17,41 \mathrm{bc}$ & $22,99 \mathrm{a}$ & $20,64 \mathrm{ab}$ & $22,37 \mathrm{ab}$ & $22,56 \mathrm{ab}$ & $12,57 \mathrm{c}$ \\
& $(2,999)$ & $(3,352)$ & $(3,352)$ & $(3,826)$ & $(1,658)$ & $(4,048)$ & $(3,098)$ \\
\hline \multirow{2}{*}{ Triptamina } & $0,87 \mathrm{~b}$ & $1,38 \mathrm{~b}$ & $1,76 \mathrm{~b}$ & $2,59 \mathrm{~b}$ & $0,09 \mathrm{~b}$ & $2,62 \mathrm{~b}$ & $12,07 \mathrm{a}$ \\
& $(0,934)$ & $(0,744)$ & $(0,626)$ & $(1,595)$ & $(0,049)$ & $(3,332)$ & $(5,957)$ \\
\hline \multirow{2}{*}{ Feniletilamina } & $0,88 \mathrm{ab}$ & $0,15 \mathrm{c}$ & $0,55 \mathrm{bc}$ & $1,16 \mathrm{a}$ & $0,02 \mathrm{c}$ & $0,84 \mathrm{~b}$ & $0,23 \mathrm{c}$ \\
& $(0,035)$ & $(0,007)$ & $(0,017)$ & $(0,641)$ & $(0,002)$ & $(0,003)$ & $(0,052)$ \\
\hline \multirow{2}{*}{ Serotonina } & $1,29 \mathrm{ab}$ & $0,62 \mathrm{~b}$ & $0,86 \mathrm{ab}$ & $0,68 \mathrm{ab}$ & $1,32 \mathrm{ab}$ & $3,10 \mathrm{ab}$ & $1,89 \mathrm{ab}$ \\
& $(0,137)$ & $(0,325)$ & $(0,093)$ & $(0,167)$ & $(0,895)$ & $(0,906)$ & $(1,008)$ \\
\hline \multirow{2}{*}{ Total } & $0,00 \mathrm{~b}$ & $0,48 \mathrm{a}$ & $0,00 \mathrm{~b}$ & $0,00 \mathrm{~b}$ & $0,00 \mathrm{~b}$ & $0,00 \mathrm{~b}$ & $0,00 \mathrm{~b}$ \\
& $(0,000)$ & $(0,792)$ & $(0,000)$ & $(0,000)$ & $(0,000)$ & $(0,000)$ & $(0,000)$ \\
\hline n 6. Val & $28,33 \mathrm{~d}$ & $38,63 \mathrm{bcd}$ & $51,99 \mathrm{ab}$ & $43,14 \mathrm{abcd}$ & $45,19 \mathrm{abc}$ & $53,27 \mathrm{a}$ & $36,90 \mathrm{~cd}$ \\
& $(7,357)$ & $(9,912)$ & $(1,902)$ & $(9,288)$ & $(2,659)$ & $(11,71)$ & $(13,88)$ \\
\hline
\end{tabular}

$\mathrm{n}=6$. Valores médios com letras distintas na linha diferem entre si pelo teste Tukey $(\mathrm{P}<0,05)$.

Os teores das poliaminas espermina e espermidina foram menores que os encontrados em carne suína fresca (Vasconcelos-Neto, 2003), porém semelhantes aos detectados em produtos cárneos similares (Eerola et al., 1997; Hernández-Jover et al., 1997a; Montel et al., 1999; Suzzi e Gardini, 2003). Essas aminas estão naturalmente presentes na carne fresca, entretanto, os teores podem ter diminuído ao longo do processamento e maturação, podendo ter sido utilizadas por microrganismos presentes como fonte de nitrogênio (Glória, 2005).

Os teores das aminas biogênicas putrescina, cadaverina, tiramina, histamina, feniletilamina, triptamina e agmatina variaram em faixas similares às detectadas por vários autores em diversos tipos de salames (Eerola et al., 1997; Hernández-Jover et al., 1997a; Montel et al., 1999; Suzzi e Gardini, 2003; Coisson et al., 2004). A serotonina foi detectada apenas na marca B.

Durante a produção de salame, as condições são favoráveis à formação de aminas biogênicas. Há um certo grau de proteólise provocada por enzimas endógenas e microrganismos, liberando aminoácidos, o que favorece a atividade descarboxilase. De acordo com Hernández-Jover et al. (1997a) e Bover-Cid et al. (2000a,b), a presença de cadaverina tem sido relacionada a microbiota deteriorante como as enterobactérias, que são lisina descarboxilase positivas. A histamina pode ser formada principalmente por enterobactérias, dentre elas, Klebsiella oxytoca, Enterobacter aerogenes e E. cloacae (Glória, 2005).

A adição de cultura iniciadora tem sido uma alternativa viável para impedir o crescimento de bactérias contaminantes e direcionar a microbiota predominante, tendo sido importante na redução dos teores de aminas biogênicas em produtos cárneos (Hernández-Jover et al., 1997b; Ayhan et al., 1999; Bover-Cid et al., 2000b; Suzzi e Gardini, 2003). Conforme descrito nos rótulos, os produtos das marcas $D$ e $G$ foram adicionados de cultura cárnea e cultivo bacteriano liofilizado, respectivamente. Essas culturas, entretanto, não se mostraram benéficas 
na redução dos teores de aminas nos produtos. Além disso, não evitaram a formação de tiramina, histamina e putrescina, havendo apenas uma redução nos teores de cadaverina. Esses resultados sugerem que a adição da cultura iniciadora apenas inibiu as bactérias lisinadescarboxilase positivas, ou seja, as enterobactérias (Bover-Cid et al., 2000b).

As culturas iniciadoras são geralmente bactérias ácido láticas e, embora não patogênicas, podem produzir aminas biogênicas. Tiramina pode ser produzida por algumas cepas de Lactococcus, Leuconostoc, Lactobacillus, Enterococcus e Carnobacterium (Montel et al., 1999; Bover-Cid et al., 2000b; Suzzi e Gardini, 2003). Algumas cepas de Lactobacillus e Leuconostoc podem também produzir histamina (Suzzi e Gardini, 2003). A produção de feniletilamina já foi observada por cepas de Enterococcus (Bover-Cid et al., 2000b). Assim sendo, antes de se utilizar cultura iniciadora, o potencial em formar e metabolizar aminas biogênicas deve ser investigado (Suzzi e Gardini, 2003).

Considerando o nível tóxico da tiramina de $10 \mathrm{mg} / 100 \mathrm{~g}, 100 \%$ das amostras poderiam causar intoxicação em indivíduos sensíveis, e crise hipertensiva em indivíduos em tratamento com drogas inibidoras da monoaminoxidase, se consumidas em quantidades superiores a 100g/dia (Fuzikawa et al., 1999). Em relação à histamina, cujo nível tóxico é de $10 \mathrm{mg} / 100 \mathrm{~g}$ (Glória, 2005), apenas salames da marca G seriam potencialmente tóxicos. Com relação a feniletilamina, cujo nível tóxico é de $3 \mathrm{mg} / 100 \mathrm{~g}$ (Glória, 2005), apenas amostras da marca $F$ poderiam causar intoxicação, com sintomas similares aos da tiramina.

A putrescina e a cadaverina podem causar sabores desagradáveis, potencializar o efeito tóxico da histamina e da tiramina, e formar, na presença de nitrito, nitrosaminas, que são potencialmente cancerígenas (Ayhan et al., 1999; Glória, 2005). Algumas indústrias já estão conseguindo minimizar os teores de aminas biogênicas, mostrando que é possível obter produtos com menores teores desses compostos.

À semelhança do observado com relação às aminas (Tab. 2 e 3), os resultados das análises físico-químicas variaram significativamente entre marcas e entre amostras de uma mesma marca, sendo estes semelhantes aos valores relatados na literatura (Chasco et al., 1993; Novelli et al., 1998; Coisson et al., 2004; Moretti et al., 2004). Essa variação é esperada uma vez que os ingredientes, e, possivelmente, suas proporções e as etapas dos processos tecnológicos variaram em função da indústria.

Tabela 2. Atividade de água e teores de umidade, proteínas, lipídios e açucares totais em diferentes marcas de salame tipo italiano comercializadas em Belo Horizonte, MG, no período de agosto de 2001 a maio de 2002

\begin{tabular}{lccccc}
\hline \multirow{2}{*}{ Marca } & Atividade & \multicolumn{4}{c}{ Teor médio (desvio-padrão) em g/100g } \\
\cline { 3 - 6 } & de água & Umidade & Proteína & Lipídio & Açúcares totais \\
\hline \multirow{2}{*}{$\mathrm{A}$} & $0,80 \mathrm{c}$ & $34,27 \mathrm{~cd}$ & $\mathbf{2 2 , 6 1} \mathbf{b}$ & $30,52 \mathrm{a}$ & $\mathbf{2 , 2 9}$ ab \\
& $(0,001)$ & $(0,993)$ & $(2,561)$ & $(1,095)$ & $(0,012)$ \\
\hline \multirow{2}{*}{$\mathrm{B}$} & $0,84 \mathrm{bc}$ & $32,49 \mathrm{~d}$ & $27,86 \mathrm{a}$ & $27,04 \mathrm{~b}$ & $\mathbf{2 , 3 3} \mathbf{a b}$ \\
& $(0,014)$ & $(2,640)$ & $(1,444)$ & $(1,274)$ & $(0,029)$ \\
\hline \multirow{2}{*}{$\mathrm{C}$} & $0,82 \mathrm{bc}$ & $\mathbf{3 5 , 6 7} \mathbf{c}$ & $25,33 \mathrm{ab}$ & $27,00 \mathrm{~b}$ & $\mathbf{2 , 8 8} \mathbf{a}$ \\
& $(0,013)$ & $(0,716)$ & $(1,329)$ & $(0,787)$ & $(0.180)$ \\
\hline \multirow{2}{*}{$\mathrm{D}$} & $\mathbf{0 , 9 1} \mathbf{a}$ & $\mathbf{3 6 , 0 9} \mathbf{c}$ & $27,24 \mathrm{a}$ & $24,43 \mathrm{c}$ & $0,00 \mathrm{c}$ \\
& $(0,014)$ & $(1,371)$ & $(0,869)$ & $(1,206)$ & $(0,000)$ \\
$\mathrm{E}$ & $0,82 \mathrm{bc}$ & $\mathbf{3 9 , 7 8} \mathbf{a}$ & $27,81 \mathrm{a}$ & $20,08 \mathrm{e}$ & $0,00 \mathrm{c}$ \\
& $(0,017)$ & $(0,841)$ & $(1,085)$ & $(0,528)$ & $(0,000)$ \\
\hline \multirow{2}{*}{$\mathrm{F}$} & $0,86 \mathrm{~b}$ & $\mathbf{3 7 , 0 3} \mathbf{b}$ & $25,23 \mathrm{ab}$ & $23,30 \mathrm{~d}$ & $\mathbf{2 , 1 2} \mathbf{b}$ \\
& $(0,054)$ & $(1,757)$ & $(1,630)$ & $(1,847)$ & $(0,062)$ \\
\hline \multirow{2}{*}{$\mathrm{G}$} & $0,80 \mathrm{c}$ & $\mathbf{3 6 , 1 3} \mathbf{c}$ & $27,63 \mathrm{a}$ & $25,43 \mathrm{bc}$ & $0,00 \mathrm{c}$ \\
& $(0,005)$ & $(0,573)$ & $(3,932)$ & $(1,504)$ & $(0,000)$ \\
\hline
\end{tabular}

$\mathrm{n}=6$. Valores médios com letras distintas na coluna diferem entre si pelo teste Tukey $(\mathrm{P}<0,05)$. Resultados em negrito indicam valores que não atenderam à legislação vigente. 
Tabela 3. Teores de nitratos, nitritos, cinzas e cloreto de sódio em diferentes marcas de salame tipo italiano comercializadas em Belo Horizonte, MG, no período de agosto de 2001 a maio de 2002

\begin{tabular}{lcccc}
\hline \multirow{2}{*}{ Marca } & \multicolumn{2}{c}{ Teor médio (desvio-padrão) } \\
$\mathrm{mg} / 100 \mathrm{~g}$ & Nitritos & Cinzas & $\mathrm{g} / 100 \mathrm{~g}$ \\
& Nitratos & $0,29 \mathrm{de}$ & $5,54 \mathrm{c}$ & $\mathrm{NaCl}$ \\
\hline \multirow{2}{*}{$\mathrm{A}$} & $5,40 \mathrm{~b}$ & $(0,030)$ & $(0,115)$ & $3,87 \mathrm{c}$ \\
& $(0,278)$ & $1,92 \mathrm{a}$ & $5,75 \mathrm{c}$ & $(0,114)$ \\
$\mathrm{n}$ & $14,95 \mathrm{a}$ & $(0,063)$ & $(0,329)$ & $4,54 \mathrm{~b}$ \\
& $(8,357)$ & $1,17 \mathrm{c}$ & $5,58 \mathrm{c}$ & $(0,241)$ \\
$\mathrm{n}$ & $4,52 \mathrm{~b}$ & $(0,297)$ & $(0,061)$ & $(0,71 \mathrm{c}$ \\
& $(0,468)$ & $1,54 \mathrm{~b}$ & $6,23 \mathrm{~b})$ \\
$\mathrm{D}$ & $3,70 \mathrm{~b}$ & $(0,148)$ & $(0,279)$ & $4,36 \mathrm{~b}$ \\
& $(0,975)$ & $0,25 \mathrm{de}$ & $6,16 \mathrm{~b}$ & $(0,061)$ \\
$\mathrm{E}$ & $3,67 \mathrm{~b}$ & $(0,034)$ & $(0,126)$ & $4,35 \mathrm{~b}$ \\
& $(0,137)$ & $0,41 \mathrm{~d}$ & $5,56 \mathrm{c}$ & $(0,129)$ \\
$\mathrm{F}$ & $4,42 \mathrm{~b}$ & $(0,096)$ & $(0,205)$ & $3,81 \mathrm{c}$ \\
& $(1,716)$ & $0,20 \mathrm{e}$ & $7,12 \mathrm{a}$ & $(0,079)$ \\
\hline \multirow{2}{*}{$\mathrm{G}$} & $5,49 \mathrm{~b}$ & $(0,050)$ & $(0,163)$ & $5,43 \mathrm{a}$ \\
& $(0,133)$ & $0,135)$ \\
\hline
\end{tabular}

$\mathrm{n}=6$. Valores médios com letras distintas na coluna diferem entre si pelo teste Tukey $(\mathrm{P}<0,05)$.

Não foi constatada correlação significativa entre teor de umidade e atividade de água nos salames, provavelmente devido ao uso de ingredientes com propriedades umectantes em diferentes proporções, como sal, açúcar, maltodextrina e leite em pó. Segundo Rao (1997), dentre os tipos de umectantes utilizados na indústria cárnea, tem-se os polióis (glicerol, propileno glicol, polietileno glicol 400, 1,3-butileno glicol e sorbitol), os açúcares (glicose, frutose, sacarose e lactose) e os sais (cloreto de sódio e de potássio), sendo o cloreto de sódio o principal redutor da atividade de água.

A maioria das amostras (85\%) estava de acordo com o limite máximo de atividade de água de 0,90 estipulado para o salame tipo italiano. Entretanto, apenas $29 \%$ atenderam ao limite máximo para o teor de umidade de $35 \mathrm{mg} / 100 \mathrm{~g}$ (Instrução..., 2000). Dentre as amostras analisadas, apenas a marca D seria susceptível ao crescimento de algumas bactérias dos gêneros Escherichia, Salmonella, Clostridium, Bacillus, Enterobacter, Citrobacter e Serratia (Rao, 1997), podendo constituir um risco à qualidade e segurança do produto.

O açúcar foi declarado como ingrediente opcional em $43 \%$ das amostras analisadas (A, C e E). Entretanto, em uma amostra na qual foi declarada a adição de açúcar, a presença desse não foi detectada. Isso pode ter acontecido pelo fato de esses serem utilizados pelas culturas iniciadoras. Os açúcares redutores foram os predominantes, correspondendo de 93 a $100 \%$ do total. Não foi encontrada correlação significativa entre os teores de açúcar e a vida de prateleira do produto no momento da análise. Não foram encontrados na literatura dados de teores de açúcares totais em salames.

Considerando o limite mínimo de $25 \mathrm{~g} / 100 \mathrm{~g}$ para proteínas e os limites máximos de $32 \mathrm{~g} / 100 \mathrm{~g}$ para lipídios e de 1,5g/100g para açucares totais para salames tipo italiano, $86 \%, 100 \%$ e $57 \%$ das amostras atenderam à legislação vigente (Instrução..., 2000) quanto a estes parâmetros analisando-os separadamente. $\mathrm{O}$ baixo atendimento à legislação em relação ao açúcar pode ser reflexo da própria metodologia analítica, que, apesar de clássica, é pouco precisa, principalmente para pequenas quantidades de açúcares, e tem-se dificuldade na visualização do ponto final da titulação. Além disso, é susceptível a interferências, inclusive pela presença de polióis, que podem ser usados como umectantes.

Os teores de nitrato e nitrito de sódio, cinzas e cloretos encontrados nas amostras são apresentados na Tab. 3. Os nitratos foram predominantes em relação aos nitritos, o que confirma a preferência de adição do nitrato devido ao seu efeito prolongado, condizente com a vida de prateleira dos produtos. Os teores encontrados são similares aos relatados por 
Novelli et al. (1998). Todas as amostras analisadas apresentaram-se dentro dos limites residuais máximos para nitrato e nitrito de sódio em carnes e produtos derivados de 30 e $15 \mathrm{mg} / 100 \mathrm{~g}$, respectivamente (Portaria..., 1998).

Os teores médios de cinzas e de cloretos, em $\mathrm{NaCl}$, variaram significativamente entre amostras e entre marcas, com valores similares aos relatados na literatura (Chasco et al., 1993; Novelli et al., 1998; Coisson et al., 2004). Os teores de cloretos encontrados representaram de 66 a 79\% dos teores de cinzas, o que reflete na correlação significativa entre os teores de cinzas e de cloretos $\left(R^{2}=0,8493, p<0,001\right)$. Limites para esses dois parâmetros não estão previstos pela legislação brasileira. De acordo com Suzzi e Gardini (2003), concentrações de $\mathrm{NaCl}$ de 3,5 a $5,5 \%$ podem inibir a formação de histamina, o que pode ter colaborado para os baixos teores de histamina encontrados.
Os valores de $\mathrm{pH}$, acidez e os índices de peróxidos obtidos para as amostras analisadas, assim como o número de dias decorridos entre a fabricação e a análise, estão apresentados na Tab. 4. Os valores médios de $\mathrm{pH}$ variaram significativamente com valores semelhantes aos descritos na literatura (Terra et al., 1987; Chasco et al., 1993; Rao, 1997; Novelli et al., 1998). A acidez titulável na gordura variou de 16,61 a $55,03 \mathrm{ml}$ de $\mathrm{NaOH} \mathrm{N} / 100 \mathrm{~g}$, não tendo sido encontrados dados para esse parâmetro na literatura consultada. Alguns autores relataram que, em valores de $\mathrm{pH}$ baixos, há produção de aminas biogênicas pelos microrganismos como proteção ao $\mathrm{pH}$ ácido. Entretanto, a inexistência de correlação significativa entre os valores de $\mathrm{pH}$ e os teores de aminas biogênicas nesse estudo reafirma que a velocidade de redução do $\mathrm{pH}$ é mais relevante do que o $\mathrm{pH}$ final na formação destas aminas (Suzzi e Gardini, 2003).

Tabela 4. Dias após a fabricação em que foram feitas as análises, valores de $\mathrm{pH}$, acidez na gordura e índice de peróxido de diferentes marcas de salame tipo italiano comercializadas em Belo Horizonte, $\mathrm{MG}$, no período de agosto de 2001 a maio de 2002

\begin{tabular}{|c|c|c|c|c|}
\hline \multirow[b]{2}{*}{ Marca } & \multirow{2}{*}{$\begin{array}{l}\text { Dias após } \\
\text { fabricação }\end{array}$} & \multicolumn{3}{|c|}{ Valor médio (desvio-padrão) } \\
\hline & & $\mathrm{pH}$ & $\begin{array}{c}\text { Acidez } \\
(\mathrm{ml} \mathrm{NaOH} \mathrm{N} / 100 \mathrm{~g})\end{array}$ & $\begin{array}{c}\text { Indice Peróxido } \\
(\mathrm{mEq} / \mathrm{kg})\end{array}$ \\
\hline A & 38 & $\begin{array}{c}5,60 \mathrm{a} \\
(0,192)\end{array}$ & $\begin{array}{l}37,25 \mathrm{~b} \\
(1,511)\end{array}$ & $\begin{array}{c}5,73 \mathrm{~b} \\
(1,095)\end{array}$ \\
\hline B & 118 & $\begin{array}{c}4,86 \mathrm{c} \\
(0,415)\end{array}$ & $\begin{array}{l}36,29 b \\
(3,795)\end{array}$ & $\begin{array}{c}334,82 \mathrm{a} \\
(1,274)\end{array}$ \\
\hline $\mathrm{C}$ & 50 & $\begin{array}{c}5,78 \mathrm{a} \\
(0,110)\end{array}$ & $\begin{array}{l}28,82 \mathrm{c} \\
(0,891)\end{array}$ & $\begin{array}{l}4,99 \mathrm{~b} \\
(0.787)\end{array}$ \\
\hline $\mathrm{D}$ & 21 & $\begin{array}{l}5,10 \mathrm{bc} \\
(0,123)\end{array}$ & $\begin{array}{l}16,61 \mathrm{~d} \\
(1,070)\end{array}$ & $\begin{array}{l}11,89 b \\
(1,206)\end{array}$ \\
\hline $\mathrm{E}$ & 33 & $\begin{array}{l}5,37 \mathrm{ab} \\
(0,129)\end{array}$ & $\begin{array}{l}26,40 \mathrm{c} \\
(1,034)\end{array}$ & $\begin{array}{l}11,58 b \\
(0,528)\end{array}$ \\
\hline $\mathrm{F}$ & 68 & $\begin{array}{l}5,16 \mathrm{bc} \\
(0,310)\end{array}$ & $\begin{array}{l}27,03 \mathrm{c} \\
(2,117)\end{array}$ & $\begin{array}{l}39,86 \mathrm{~b} \\
(1,847)\end{array}$ \\
\hline G & 49 & $\begin{array}{c}5,77 \mathrm{a} \\
(0,256) \\
\end{array}$ & $\begin{array}{l}55,03 \text { a } \\
(9,436)\end{array}$ & $\begin{array}{c}0,00 \mathrm{~b} \\
(1,504)\end{array}$ \\
\hline
\end{tabular}

$\mathrm{n}=6$. Valores médios com letras distintas na coluna diferem entre si pelo teste Tukey $(\mathrm{P}<0,05)$.

Com relação ao índice de peróxido, uma amostra apresentou valores significativamente maiores que as demais. Essa amostra era a que possuía o maior tempo de fabricação, sugerindo a influência do tempo de armazenamento. Entretanto, segundo Terra et al. (1987), os índices de peróxido atingiram valores máximos do $3^{\circ}$ ao $10^{\circ}$ dias após a fabricação de salames tipo italiano, decaindo posteriormente. Limites para $\mathrm{pH}$, acidez titulável e índice de peróxidos não estão previstos pela legislação brasileira.

Estudos de correlação de Pearson indicaram correlação positiva significativa, em nível de $1 \%$ de significância, do teor total de aminas com os teores de tiramina $\left(\mathrm{R}^{2}=0,91\right)$ e putrescina 
$\left(R^{2}=0,75\right)$, sugerindo que essas são as aminas que mais contribuem ao teor total que se acumula no salame. Apesar de relatada em alguns estudos, não foi observada correlação significativa em nível de $1 \%$ de probabilidade entre os teores totais de aminas e as características físicoquímicas. Isso reafirma a complexidade do fenômeno de formação de aminas e sua dependência simultânea de vários fatores.

\section{CONCLUSÕES}

É preocupante o fato de os teores de tiramina encontrados em todas as marcas serem potencialmente capazes de causar intoxicação. Amostras de duas marcas poderiam causar também intoxicação por histamina e por feniletilamina. Nenhuma das marcas analisadas estava em conformidade com a legislação brasileira no que diz respeito a, pelo menos, uma das características físico-químicas de identidade e qualidade. Sugere-se a inclusão de tiramina como característica para a garantia da qualidade de salames tipo italiano e que as indústrias envidem esforços para minimizar os teores de aminas biogênicas nos salames tipo italiano e se adequem à legislação vigente.

\section{AGRADECIMENTOS}

E. Tavares e M.M. Araújo pela colaboração a este trabalho.

\section{REFERÊNCIAS BIBLIOGRÁFICAS}

AYHAN, K.; KOLSARICI, N.; OZKAN, G.A. The effects of a starter culture on the formation of biogenic amines in Turkish soudjoucks. Meat Sci., v.53, p.183-188, 1999.

BOVER-CID, S.; IZQUIERDO-PULIDO, M.; VIDAL-CAROU, M.C. Influence of hygienic quality of raw material on biogenic amine production during ripening and storage of dry fermented sausages. J. Food Protec., v.63, p.1544-1550, 2000a.

BOVER-CID, S.; IZQUIERDO-PULIDO, M.; VIDAL-CAROU, M.C. Mixed starter cultures to control biogenic amine production in dry fermented sausages. J. Food Protec., v.63, p.1556-1562, 2000b.

CHASCO, J.; BERIAIN, M.J.; BELLO, J. A study of changes in the fat content of some varieties of dry sausage during curing. Meat Sci., v.34, p.191-204, 1993.

COISSON, J.D.; CERUTTI, C.; TRAVAGLIA, F. et al. Production of biogenic amines in "Salamini italiani alla cacciatora PDO". Meat Sci., v.67, p.343-349, 2004.

EEROLA, S.; ROIG-SAGUÉS, A.X.; LILLEBERG, L. et al. Biogenic amines in dry sausages during shelf-life storage. Z. Lebensm. Unters. Forsch., v.205, p.351-355, 1997.

ELLIOT, R.J.; PORTER, A.G. A rapid cadmium method for the determination of nitrate in bacon and curing brines. In: AFNOR, v.4, p.410, 1974.

FUZIKAWA, C.S.; HARA, C.; GLÓRIA, M.B.A. et al. IMAO e dieta - atualização e orientações práticas para o uso clínico. J. Bras. Psiq., v.48, p.453-460, 1999.

GLÓRIA, M.B.A. Amines. In: HUI, H.; NOLLET, L.L. Handbook of Food Science. New York, Marcel Dekker, 2005.Cap.13. p.38

HERNÁNDEZ-JOVER, T.; IZQUIERDOPULIDO, M.; VECIANA-NOGUÉS, M.T. et al. Biogenic amine and polyamine contents in meat and meat products. J. Agric. Food Chem., v.45, n.6, p.2098-2102, 1997a.

HERNÁNDEZ-JOVER, T.; IZQUIERDOPULIDO, M.; VECIANA-NOGUÉS, M.T. et al. Effect of starter cultures on biogenic amine formation during fermented sausage production. J. Food Protec., v.60, p.825-830, 1997 b.

INSTRUÇÃO Normativa $n^{\circ} 20$ de 21 de julho de 1999, SDA/DIPOA. Oficializar os Métodos Analíticos Físico-químicos para Controle de Produtos Cárneos e Ingredientes. Disponível em $<$ http://www.agricultura.gov.br/sda/dipoa/instnor m20.html. Acessado em: 07/09/2005.

INSTRUÇÃO Normativa ${ }^{\circ} 22$ de 31 de julho de 2000, SDA/DIPOA. Aprovar os Regulamentos Técnicos de Identidade e Qualidade. Disponível em <http://www.agricultura.gov.br/sda/dipoa/ instnorm22_2000.htm. Acessado em: 07/09/2005.

LEITÃO, M.F.F.; BALDINI, V.L.S.; SALES, A.M. Histamina em pescado e alimentos 
industrializados. Col. ITAL, v.13, p.123-130, 1983.

MONTEL, M.C.; MASSON, F.; TALON, R. Comparison of biogenic amine content in traditional and industrial French dry sausages. Sci. Alim., v.19, n.2, p.247-254, 1999.

MORETTI, V.M.; MADONIA, G.; DIAFERIA, C. et al. Chemical and microbiological parameters and sensory atributes of a typical Sicilian salami ripened in different conditions. Meat Sci., v.66, p.845-854, 2004.

NORMAS analíticas: métodos químicos e físicos para análise de alimentos. São Paulo: Intituto Adolfo Lutz, 1985. p.340-344.

NOVELLI, E.; ZANARDI, E.; GHIRETTI, G.P. et al. Lipid and cholesterol oxidation in frozen stored pork, salame milano and mortadella. Meat Sci., v.48, p.29-40, 1998.

PORTARIA $\mathrm{n}^{0} 1.004$ de 11 de dezembro de 1998, SDA/DIPOA.. Aprovar o Regulamento Técnico: "Atribuição de Função de Aditivos e seus Limites Máximos de uso para a Categoria 8 - Carnes e Produtos Cárneos". Disponível em
$<$ http://www.anvisa.gov.br/legis/portarias/1002 98.htm. Acessado em: 07/09/2005.

RAO, D.N. Intermediate moisture foods based on meats - a review. Food Rev. Int., v.13, p.519$551,1997$.

SILVA, C.M.G.; GLÓRIA, M.B.A. Bioactive amines in chicken breast and thigh after slaughter and during storage at $4 \pm 1{ }^{\circ} \mathrm{C}$ and in chicken-based meat products. Food Chem., v.78, p.241-248, 2002.

SUZZI, G.; GARDINI, F. Biogenic amines in dry fermented sausages: a review. Int. J. Food Microbiol., v.88, p.41-54, 2003.

TERRA, N.N.; FROZZI, V.; STOFFLS, I. et al. Efeitos secundários do glutamato monossódico no salame tipo italiano. Rev. Nac. Carne, v.11, p.12-13, 1987.

VASCONCELOS-NETO, M.C. Características físico-químicas, microbiológicas e aminas bioativas em carne suína. 2003, 70f. Dissertação (Mestrado em Ciência de Alimentos) - Faculdade de Farmácia, Universidade Federal de Minas Gerais, Belo Horizonte. 